\title{
NOUVELLE
}

\section{Le microbiote intestinal : clé de voûte entre l'obésité maternelle et les troubles de la socialisation chez la descendance}

Anne-Laure Barbotin ${ }^{1,2}$, Paolo Giacobini ${ }^{1}$, Vincent Prévot ${ }^{1}$
${ }^{1}$ Unité Inserm U1172, laboratoire du développement et plasticité du cerveau neuroendocrine, centre de recherche Jean-Pierre Aubert, 1, place de Verdun, 59045 Lille, France ; ${ }^{2}$ Institut de Biologie de la Reproduction-Spermiologie-CECOS, CHU de Lille, avenue Eugène Avinée, 59037 Lille, France. vincent.prevot@inserm.fr anne-laure.barbotin@chru-lille.fr
> Le microbiote intestinal est un écosystème composé de micro-organismes (essentiellement des bactéries) vivant en symbiose avec leur hôte [1]. Il a été mis en évidence que sa composition pouvait varier selon le type de régime alimentaire [2]. Des études récentes ont révélé que le microbiote exerçait des effets $(\rightarrow)$ Voir la nouvelle physiologiques et de S. Normand et al., qu'un déséquilibre $m / s n^{\circ}$ 6-7, juin-juillet de celui-ci appelé dysbiose [12] $(\rightarrow)$ pouvait être impliqué dans certaines pathologies comme l'obésité [13] $(\rightarrow)$ ou l'autisme $[3,4$, 14] $(\rightarrow)$. 2013, page 586

$(\rightarrow)$ Voir la Synthèse de L. Genser et al., $\mathrm{m} / \mathrm{s} \mathrm{n}{ }^{\circ} 5$, mai 2016, page 461

$(\rightarrow)$ Voir la Synthèse de A. El Kaoutari et al., $m / s n^{\circ} 3$, mars 2014, page 259

II semble également exister un lien entre l'obésité maternelle et la survenue d'anomalies du neuro-développement incluant notamment les troubles du spectre autistique (TSA) chez l'enfant [5].

Une étude récente menée par Buffington et ses collègues publiée dans Cell [6] rapporte en effet qu'un régime riche en graisses entraînant une obésité chez la souris gestante était associé à des troubles du comportement en rapport avec une modification du microbiote chez la descendance (Figure 1).

Influence du régime alimentaire maternel et du microbiote sur les comportements sociaux de la descendance

Dans un premier temps, les auteurs ont étudié l'influence du régime alimentaire maternel sur le neuro-développement de la descendance. Pour cela, des souris femelles ont été soumises à un régime hyperlipidique (high fat diet, ou HFD) obésogène en comparaison à des souris soumises à un régime classique pendant 8 semaines avant la gestation. La progéniture de toutes les souris a été sevrée à 3 semaines puis soumise à un régime alimentaire standard. Les facultés de socialisation des souriceaux ont été étudiées après 4 semaines à l'aide d'une étude comportementale en cage ${ }^{l}$. Les résultats montrent que les petits issus de femelles ayant reçu un régime riche en graisses se révèlent moins sociables que ceux issus de mères ayant reçu un régime alimentaire standard.

\section{Régime hyperlipidique maternel et modification du microbiote de la} descendance

Afin d'examiner un lien causal entre régime hyperlipidique et dysbiose chez la progéniture, la composition bactérienne intestinale des souriceaux a été évaluée grâce au séquençage de l'ARN 16S contenu dans leurs matières fécales

$(\rightarrow)$ Voir la Synthèse de J. Weissenbach et A. Sghir, page 937 de ce numéro
Cette analyse révèle que le microbiote des petits issus de mères soumises au régime hyperlipidique est moins diversifié, confirmant ainsi que la dysbiose maternelle induite par le type de régime se transmet à la descendance.

Bien que la composition du microbiote soit propre à chaque individu, les souris étant coprophages, le microbiote des souris partageant une même cage se transfère d'un individu à l'autre. Ainsi, afin de déterminer si la survenue des troubles du comportement pouvait être évitée en rééquilibrant le microbiote, des expériences de cohabitation entre la progéniture des souris femelles traitées par les deux types de régime ont été réalisées. Les résultats montrent que la cohabitation restaure un profil phylogénétique équilibré du microbiote chez les petits issus de souris soumises au régime hyperlipidique. D'autre part, il s'avère que la cohabitation des souriceaux prévient aussi l'apparition des troubles de la socialisation.

Cependant, les auteurs ont montré, lors d'expériences de transfert du microbiote fécal (issu de la progéniture des souris soumises au régime hyperlipidique ou au régime classique) à des souris axéniques (germ free), qu'il existait une fenêtre neuro-développementale durant laquelle la reconstitution du microbiote améliorait significativement le comportement social. $\varepsilon$ n effet, seules les souris ayant reçu le microbiote fécal (issu de la descendance des souris soumises au régime classique) 4
${ }^{1}$ L'animal étudié est placé au centre d'une cage à 3 compartiments et est libre de se déplacer soit vers les compartiments vides, soit vers celui contenant une autre souris, ainsi il est possible de mesurer le temps passé dans chaque compartiment et donc la préférence pour l'interaction sociale [7]. 


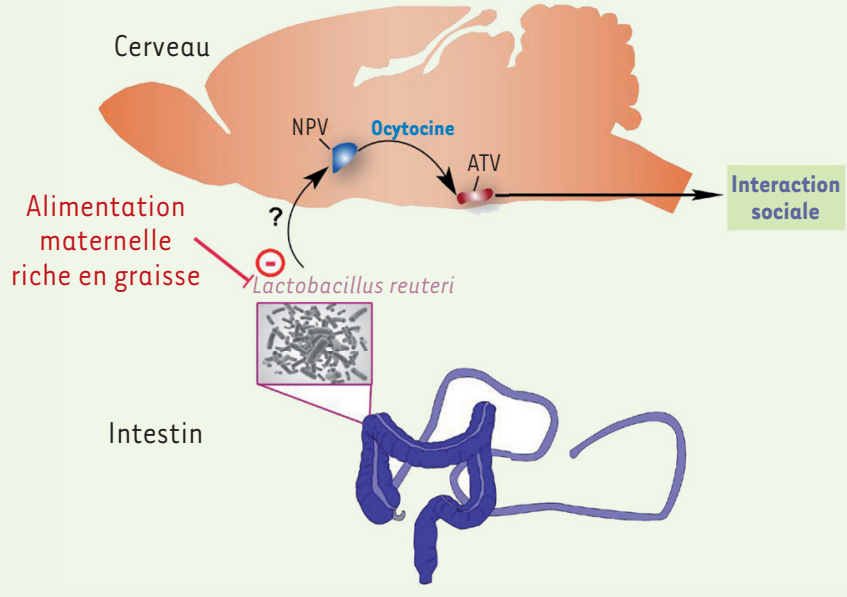

Figure 1. Régime riche en graisses et troubles du comportement. D'après une récente étude publiée dans Cell [6], les troubles de socialisation observés dans la descendance des souris soumises à un régime hyperlipidique pendant la gestation et la période d'allaitement semblent impliquer une perturbation de la flore intestinale qui entraînerait une altération de la communication intestincerveau dont les acteurs principaux seraient : la souche bactérienne Lactobacillus reuteri, les neurones à ocytocine du noyau paraventriculaire hypothalamique (NPV) et les neurones à dopamine de l'aire tegmentale ventrale (ATV). Néanmoins, le mode d'action de la souche bactérienne sur le cerveau reste inconnu et l'implication des neurones à ocytocine inférentielle.

semaines après la naissance (au moment du sevrage) montrent une socialisation normale tandis que l'administration plus tardive du microbiote (à 8 semaines) ne modifie pas leur comportement.

\section{Une bactérie identifiée : Lactobacillus reuteri}

Une analyse métagénomique des matières fécales des souriceaux révèle que la quantité de certaines espèces est particulièrement réduite dans le microbiote de la progéniture des souris soumises au régime hyperlipidique. Parmi celles-ci, Lactobacillus reuteri s'avère être l'espèce la plus significativement touchée, avec une concentration 9 fois moins importante par rapport à celle observée dans le microbiote de la population de référence. Les auteurs émettent alors l'hypothèse que si le déficit en $L$. reuteri est responsable de l'apparition de troubles du comportement et de la socialisation, sa réintroduction dans le microbiote pourrait alors corriger ces symptômes. Afin de tester cette hypothèse, $L$. reuteri a été ajoutée à l'eau mise à disposition des souriceaux au moment du sevrage, ceci pendant 4 semaines. Parmi les souris issues des mères soumises au régime hyperlipidique, celles ayant reçu l'eau enrichie en $L$. reuteri montrent une meilleure socialisation. De plus, alors que l'administration de la souche vivante de L. reuteri améliore la socialisation des souris, l'introduction d'une souche inactive (tuée par la chaleur) ou d'une autre espèce de Lactobacillus ne modifie pas le comportement, confirmant donc la spécificité de L. reuteri pour empêcher l'apparition des troubles.

Cependant, l'amélioration du comportement ne concerne pas tous les symptômes rencontrés dans le TSA. En effet, il semblerait que le microbiote intestinal n'ait pas d'influence sur les comportements répétitifs, mais améliore uniquement les symptômes en rapport avec des déficits de la communication et de l'interaction sociale.

\section{Mécanisme d'action de L. reuteri}

En 2013, Poutahidis et al. ont montré que L. reuteri augmentait les taux d'ocytocine
[8]. Or l'ocytocine, parfois appelée « hormone de l'attachement », est connue pour jouer un rôle clé dans les comportements sociaux [9] mais aussi pour son implication dans les syndromes autistiques [10]. L'amélioration des symptômes par L. reuteri serait donc liée à une augmentation de cette hormone. L'ocytocine étant sécrétée par des neurones situés au niveau du noyau paraventriculaire (NPV) de l'hypothalamus, cette hypothèse a donc été testée en comparant le nombre de cellules exprimant l'ocytocine au niveau du NPV. Les résultats montrent une diminution du nombre des neurones immunoréactifs pour l'ocytocine, chez les souriceaux issus des souris soumises au régime hyperlipidique par rapport à ceux issus de souris ayant reçu un régime standard ; cette différence constatée n'étant pas imputable à une diminution globale de la quantité de neurones, celle-ci étant similaire entre les deux groupes. De plus, cette immunoréactivité diminuée a pu être corrigée grâce à la réintroduction de L. reuteri.

Les neurones à ocytocine du NPV se projettent dans l'aire tegmentale ventrale (ATV). Cette région cérébrale est notamment connue pour appartenir au système de la récompense mésolimbique dopaminergique et est donc impliquée dans les comportements sociaux. L'ocytocine active les neurones dopaminergiques situés dans l'ATV chez la souris et chez l'homme, facilitant les processus de socialisation. Partant du postulat qu'une interaction sociale peut activer le système de récompense dopaminergique via le déclenchement de potentiels d'action synaptiques au niveau des neurones de I'ATV, les auteurs ont procédé à une étude électrophysiologique comparative de la potentialisation à long terme (PTL) provoquée par les interactions sociales. Les résultats de cette expérience montrent que la nouveauté sociale (rencontre de deux souris non familières) provoque une PTL au niveau des neurones dopaminergiques chez les souris contrôles alors qu'aucun changement n'est constaté chez les souris issues des femelles sou- 
mises au régime hyperlipidique. Les interactions sociales activent donc bien le système de récompense mésolimbique chez les souris contrôles.

De plus, les auteurs ont mis en évidence qu'une PTL similaire à celle observée chez les souris contrôles pouvait être restaurée grâce au traitement par L. reuteri. Le mécanisme d'action de $L$. reuteri semblant lié à l'augmentation des taux d'ocytocine, les auteurs ont donc testé l'effet d'un traitement directement basé sur l'administration d'ocytocine pour corriger les troubles du comportement. Alors que l'utilisation d'ocytocine seule par voie intra-nasale n'entraîne pas de PTL, l'association d'ocytocine combinée à une interaction sociale permet de restaurer l'activation neuronale, suggérant l'existence d'un effet synergique nécessaire de l'ocytocine et de la dopamine dans le processus de socialisation.

Ainsi, les troubles autistiques observés dans la descendance des souris soumises à un régime hyperlipidique semblent impliquer les neurones à ocytocine et les connexions synaptiques qu'ils effectuent au niveau de l'ATV.

\section{Conclusion et perspectives}

Ces résultats offrent une vision nouvelle du mécanisme par lequel un changement de l'écosystème microbiologique causé par un régime hyperlipidique pourrait avoir un impact sur le neuro-développement de la descendance en lien avec des modifications neuronales. À l'instar d'une étude précédente ayant montré l'intérêt de l'utilisation de probiotiques dans un modèle murin présentant un syndrome autistique [11], ces récents résultats suggèrent $L$. reuteri comme candidat afin d'améliorer plus spécifiquement les troubles de la socialisation [6]. Ces résultats offrent ainsi de nouvelles perspectives thérapeutiques concernant l'intérêt des probiotiques comme traitement non-invasif potentiel. Toutefois, de futures recherches devront déterminer si ces découvertes peuvent s'appliquer à l'homme. En effet, le microbiote possède une grande résilience fonctionnelle pouvant en partie expliquer les résultats contradictoires des études concernant les liens de causalité entre microbiote et pathologies et donc sur l'intérêt de l'utilisation des probiotiques en pathologie humaine. $\diamond$ Maternal obesity alters social brain programming by altering gut microbiota in progeny

\section{LIENS D'INTÉRÊT}

Les auteurs déclarent n'avoir aucun lien d'intérêt concernant les données publiées dans cet article.

\section{RÉFÉRENCES}

1. Landman C, Quévrain $\varepsilon$. Le microbiote intestinal : description, rôle et implication physiopathologique. Rev Med Interne 2016 ; 37 : 418-23.
2. Tremaroli V, Bäckhed F. Functional interactions between the gut microbiota and host metabolism. Nature 2012 ; 489 : 242-9.

3. Galley JD, Bailey M, Kamp Dush C, et al. Maternal obesity is associated with alterations in the gut microbiome in toddlers. PLoS One 2014 ; 9 : el13026.

4. Ma J, Prince AL, Bader D, et al. High-fat maternal diet during pregnancy persistently alters the offspring microbiome in a primate model. Nat Commun 2014 ; $5: 3889$.

5. Connolly N, Anixt J, Manning P, et al. Maternal metabolic risk factors for autism spectrum disorder: An analysis of electronic medical records and linked birth data. Autism Res $2016 ; 9: 829-37$.

6. Buffington SA, Di Prisco GV, Auchtung TA, et al. Microbial reconstitution reverses maternal dietinduced social and synaptic deficits in offspring. Cell $2016 ; 165: 1762-75$.

7. Silverman JL, Yang M, Lord C, et al. Behavioural phenotyping assays for mouse models of autism. Nat Rev Neurosci 2010 ; 11 : 490-502.

8. Poutahidis T, Kearney SM, Levkovich T, et al. Microbial symbionts accelerate wound healing via the neuropeptide hormone oxytocin. PLoS One 2013 ; 8 : e78898.

9. Donaldson ZR, Young LJ. Oxytocin, vasopressin, and the neurogenetics of sociality. Science 2008 ; 322 : 900-4.

10. Lerer $\varepsilon$, Levi $S$, Salomon $S$, et al. Association between the oxytocin receptor (OXTR) gene and autism: relationship to Vineland adaptive behavior scales and cognition. Mol Psychiatry $2008 ; 13: 980-8$.

11. Hsiao EY, McBride SW, Hsien S, et al. Microbiota modulate behavioral and physiological abnormalities associated with neurodevelopmental disorders. Cell 2013 ; 155 : 1451-63.

12. Normand $S$, Secher T, Chamaillard M. La dysbiose, une nouvelle entité en médecine? Med Sci (Paris) 2013 ; $29: 586-9$.

13. Genser L, Poitou C, Brot-Laroche $\varepsilon$, et al. L'altération de la perméabilité intestinale : chaînon manquant entre dysbiose et inflammation au cours de l'obésité ? Med Sci (Paris) 2016 ; 32 : 461-9.

14. El Kaoutari A, Armougom F, Raoult D, Henrissat B. Le microbiote intestinal et la digestion des polysaccharides. Med Sci (Paris) 2014 ; $30: 259-65$.

15. Weissenbach J, Sghir A. Microbiotes et métagénomique. Med Sci (Paris) $2016 ; 32: 937-43$.

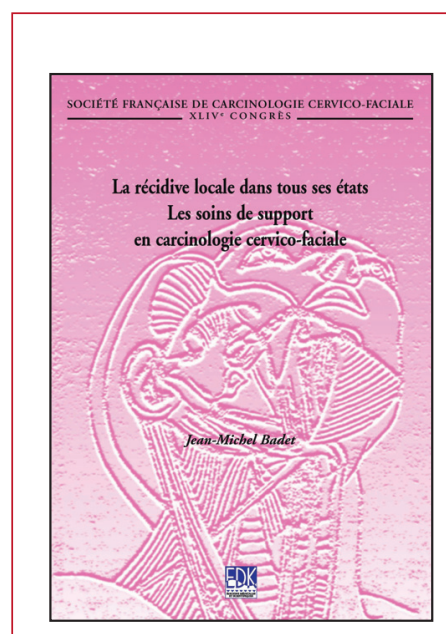

ISBN : 978-2-8425-4174-3 274 pages

\section{Bon de commande}

À retourner à EDK, 109, avenue Aristide Briand - 92541 Montrouge Cedex Tél. : 0141177405 - Fax : 0149850345 - E-mail : francois.flori@edpsciences.org

NOM : ....................................................................... Prénom :

Adresse :

Code postal :

Ville :

Pays :

Fonction :

Je souhaite recevoir l'ouvrage La récidive locale dans tous ses états - Les soins de support en carcinologie cervico-faciale : $35 €+3 €$ de port $=\mathbf{3 8} €$ TTC

en ............... exemplaire, soit un total de .................................. €
$\square$ Par chèque, à l'ordre de EDP Sciences
$\square$ Par carte bancaire : $\square$ Visa $\square$ Eurocard/Mastercard

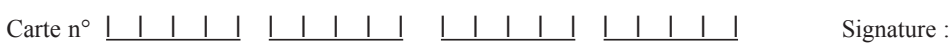

Date d'expiration: $\quad \underline{1} 1 \quad 111$

$\mathrm{N}^{\circ}$ de contrôle au dos de la carte : $\quad \underline{1 \quad|\quad|}$ 